\title{
The effect of three different energy drinks on oxygen consumption and perceived exertion during treadmill exercise
}

\author{
Gabriel J Sanders ${ }^{1 *}$, Willard Peveler ${ }^{1}$, Brady Holmer ${ }^{1}$, Corey A Peacock ${ }^{2}$ \\ From The Twelfth International Society of Sports Nutrition (ISSN) Conference and Expo \\ Austin, TX, USA. 11-13 June 2015
}

\section{Background}

Some energy drink manufacturers claim that their products can increase athletic performance. However, there are no studies to assess the effect of these energy drinks on oxygen consumption $\left(\mathrm{VO}_{2}\right)$ or ratings of perceived exertion (RPE) during exercise. If these energy drinks improve performance, $\mathrm{VO}_{2}$ and $\mathrm{RPE}$ would likely be reduced during any given exercise intensity.

\section{Methods}

Fifteen (22.1 \pm 2.7 years old) participants completed the study. Maximal oxygen consumption $\left(\mathrm{VO}_{2} \max \right)$ was initially measured to establish each participant's exercise for the $70 \%$ treadmill exercise protocol after ingesting an energy drink. Following $\mathrm{VO}_{2} \max$ testing, all participants completed a total of four conditions. Each condition required a participant to ingest an energy drink then rest in a seated position for one hour. Following one hour of rest, participants exercised for a total of 15 minutes on a treadmill at $70 \%$ of their $\mathrm{VO}_{2} \max$. For each condition, participants blindly ingested one of four price-matched beverages (12 oz. placebo (Squirt), $8.4 \mathrm{oz}$. Red Bull ${ }^{\circledR}, 16$ oz. Monster Energy ${ }^{\circledR}, 2$ oz. 5-hour ENERGY $\left.{ }^{\mathbb{R}}\right)$. Relative $\mathrm{VO}_{2}\left(\mathrm{ml} \mathrm{kg}^{-1} \cdot \mathrm{min}^{-1}\right)$ and RPE (6-20 Borg Scale) were recorded each minute during the treadmill exercise and averaged in five-minute increments and as an average for each 15-minute condition.

\section{Results}

Analysis of variance revealed there was no significant main effect of energy drinks on average $\mathrm{VO}_{2}$ (placebo $35.8 \pm$ $2.3 \mathrm{ml} . \mathrm{kg}^{-1} \cdot \mathrm{min}^{-1}$; Red Bull $35.4 \pm 2.3 \mathrm{ml} \cdot \mathrm{kg}^{-1} \cdot \mathrm{min}^{-1}$;
Monster $35.8 \pm 2.2 \mathrm{ml} . \mathrm{kg}^{-1} \mathrm{~min}^{-1}$; 5 -hour $36.5 \pm 2.4 \mathrm{ml} . \mathrm{kg}^{-}$ ${ }^{1} \mathrm{~min}^{-1} ; \mathrm{p} \geq .482$ ) and RPE (placebo $12.2 \pm 0.6$; Red Bull $12.6 \pm 0.5$; Monster $12.0 \pm 0.5$; 5-hour $11.7 \pm 0.5$; $\mathrm{p} \geq .179$ ) during 15 minutes of treadmill exercise.

\section{Conclusions}

Energy drinks do not appear to improve perceived treadmill exercise performance nor running economy assessed via oxygen consumption at $70 \%$ treadmill exercise. Given that no significant reductions were found in $\mathrm{VO}_{2}$ and RPE post energy drink consumption, results do not support manufacturers' claims regarding their product's ability to boost performance. Additional research is needed to assess time trial or time to exhaustion sprint and endurance performance. Time trials and time to exhaustion may better assess if these energy drinks can, in fact, improve exercise performance.

\section{Authors' details}

${ }^{1}$ Northern Kentucky University, Highland Heights, KY, USA. ${ }^{2}$ Nova Southeastern University, Fort Lauderdale, FL, USA.

Published: 21 September 2015

doi:10.1186/1550-2783-12-S1-P1

Cite this article as: Sanders et al:: The effect of three different energy drinks on oxygen consumption and perceived exertion during treadmill exercise. Journal of the International Society of Sports Nutrition 201512 (Suppl 1):P1.

* Correspondence: sandersg1@nku.edu

${ }^{1}$ Northern Kentucky University, Highland Heights, KY, USA

Full list of author information is available at the end of the article 